\title{
The Effect of Prior Nerve Block on Neuropathic Pain Induced by Partial Sciatic Nerve Injury in Rats
}

\author{
Takumi Nagaro ${ }^{1}$, Kazushi Takaishi ${ }^{1}$, Hitoshi Kojo ${ }^{1}$, \\ Elizabeth A. Disbrow ${ }^{2}$ and John H. Eisele ${ }^{2}$ \\ ${ }^{1}$ Department of Anesthesiology \& Resuscitology, Ehime University School of Medicine, Ehime, Japan and \\ ${ }^{2}$ Department of Anesthesiology, University of California, Davis (UCD) School of Medicine, CA, U.S.A.
}

(Received 20 January 1997, accepted 21 March 1997)

\begin{abstract}
Using the sciatic nerve partial ligation model in rats, we investigated whether prior nerve block have prophylactic effect on the development of neuropathic pain by suppressing initial nerve injury discharge. The sciatic nerve block was done with bupivacaine prior to the ligation of one half of the sciatic nerve in ten rats (bupivacaine group), and saline instead of bupivacaine was used in another ten rats as a control (saline group). The neuropathic pain related-behaviors; paw posture, and hypersensitivity to touch, heat and mechanical stimulus were compared between bupivacaine and saline groups up to 43 days after ligation. There were no statistically significant differences of pain relatedbehaviors between bupivacaine and saline groups. These results suggested that prior nerve block has no prophylactic effect on the development of neuropathic pain induced by the sciatic nerve partial ligation model in rats.
\end{abstract}

Key words : neuropathic pain, nerve block, preemptive analgesia, partial ligation model

\section{INTRODUCTION}

Neuropathic pain following peripheral nerve injury poses a serious problem because in many cases the pain is not relieved in spite of numerous therapeutic measures ${ }^{7,24)}$. The mechanism of neuropathic pain is not well understood, however, an accumulation of data suggests that neuropathic pain is related to abnormal peripheral neural input and central nervous system sensitization $^{9,19)}$. Recent studies have shown that abnormal afferent impulses from the damaged tissue or injured nerves, especially the $\mathrm{C}$-fiber afferents, induce the hypersensitivity of dorsal horn cells via NMDA receptor activation $^{8,28)}$, and it is speculated that these events 
enhance the gene expression, consequently alter the cell function and continue the state of hypersensitivity of neurons $s^{6,9,20)}$. From this it is hypothesized that injury discharge, described as massive afferent impulses during nerve injury, plays an important role in the establishment of neuropathic pain ${ }^{12,22,25,27)}$.

Three neuropathic pain models showing the pain related-behaviors observed in causalgia have been developed recently. In the chronic constrictive injury (CCI) model developed by Bennett et al. ${ }^{5)}$ four loose ligatures are applied around the entire sciatic nerve. In partial ligation (PL) model developed by Seltzer et al. ${ }^{21}$ one third to one half of the sciatic nerve is tightly ligated with a single suture through the nerve. In spinal nerve ligation (SNL) model developed by Kim et al. ${ }^{18)}$ in which one or two spinal nerves (L5 spinal nerve, or L5 and L6 spinal nerves) constituting of the sciatic nerve is tightly ligated. In these models neuropathic pain related-behaviors are observed shortly after the ligation. There are some differences in pain related-behaviors among these models. Hypersensitivity to heat is observed in both CCI and PL models, however hypersensitivity to repetitive fine touch is definitely observed in PL model, but not in CCI model, and allodynia to cold is observed in CCI model, but not in PL model ${ }^{5,21)}$. In SNL model the pain related-behaviors similar with, but smaller magnitude than PL model are observed $^{18)}$.

It is great concern whether neuropathic pain is prevented by preemptive analgesia. In CCI model, prior nerve block shortened the duration of heat hypersensitivity ${ }^{10,23)}$ or delayed the onset of heat hypersensitivity ${ }^{29)}$. In PL model Dougherty et al. showed no propylactic effect on the development of heat hypersensitivity of the prior nerve block $^{10)}$.

The efficacy of preemptive analgesia has been examined hitherto only by the hypersensitivity to heat with Hargreaves' plantar test $^{10,23,29)}$. However there is a continuing issue with use of the Hargreaves' plantar test in animal in which the foot is not resting firmly on the floor as seen in CCI and PL models in which withdrawal latency to heat (WLH) becomes shorter $^{15)}$. Furthermore, PL model shows several other pain related-behaviors than the hypersensitivity to heat ${ }^{21)}$, which may be effected differently by the prior nerve block. Bach et al. ${ }^{3)}$ suggested that the effect of prior nerve block became apparent not in an early, but in late stage. So we reevaluated the effect of prior nerve block on the development of neuropathic pain in PL model, by observing not only heat sensitivity but also several other kinds of pain related-behaviors and for more longer time than the previous report.

\section{MATERIALS AND METHODS}

A total of 30 male Sprague-Dawley rats were used, weighing between $250 \mathrm{~g}$ and $300 \mathrm{~g}$ at the start of the experiment. The study was approved by the Institutional Animal Care and Use at the University of California in Davis.

\section{Surgery}

Anesthesia was induced by inhalation with halothane $3.0 \sim 3.5 \%$, and maintained at a concentration of $1.5 \sim 2.0 \%$ with a face mask using a flow of oxygen $0.5 \sim 1.0 \mathrm{l} / \mathrm{min}$. The halothane concentration was reduced to $1.25 \sim 1.5 \%$ (1.1 $\sim 1.4 \mathrm{MAC}$ ) for 15 minutes before ligation of the sciatic nerve to reduce the blocking effect of halothane on noxious stimulation-induced central sensitization ${ }^{13)}$. The concentration of $\mathrm{CO}_{2}$ in the face mask was kept below $0.6 \%$ by adjusting the oxygen flow and the vacuum from the face mask. The body (rectal) temperature was maintained at 37.5 to $38.5^{\circ} \mathrm{C}$.

The operation was performed under sterile conditions as described by Seltzer et al. ${ }^{21)}$. The right sciatic nerve was exposed at a high level, just distal to the branch point of the posterior biceps semitendinosus nerve from the common sciatic nerve. With the aid of a microscope, a 70 silk suture on a needle was passed through the dorsal $1 / 2$ of the nerve and was tightly ligated. The wound was closed with muscle and skin sutures (4-0 silk). In preliminary studies massive whole body and right leg non-purposeful jerking movements were observed during ligation and for several seconds to one minute after ligation, which suggested that a barrage of afferent impulses were generated during and for a short 
time after ligation.

\section{Nerve block}

Under halothane anesthesia, nerve block was performed with bupivacaine prior to nerve ligation in 10 rats (bupivacaine group). Following exposure of the sciatic nerve, $0.05 \mathrm{ml}$ of $0.5 \%$ bupivacaine was injected around the exposed nerve at the site to be ligated and a small piece of sterile cotton impregnated with $0.05 \mathrm{~m} l$ of $0.5 \%$ bupivacaine was placed around the nerve. Forty minutes later, cotton was removed and the nerve was ligated. Preliminary studies consistently showed that with this technique of nerve block neither body nor leg movements were observed at the time of ligation, and the stimulation of the sciatic nerve proximal to the nerve block with $0.1 \mathrm{~ms}$ rectangular pulses (150\% supramaximal voltage) delivered at $2 \mathrm{~Hz}$ did not evoke any twitch of the gastrocnemius. Further, the right hind foot demonstrated temporary paralysis by not responding to forceps pinching until 30 to $45 \mathrm{~min}$ after sciatic ligation (20 to $35 \mathrm{~min}$ after recovery from halothane anesthesia). Saline, instead of bupivacaine, was applied to the nerve in like fashion in another 10 rats (saline group). In additional 10 rats the right sciatic nerve was exposed without drug application or nerve ligation (sham operation group).

The operations and observations were performed by a person who did not know which solution, saline or bupivacaine, was used for nerve application. The animals were studied preoperatively, and on days postoperative $1,4,8$, $15,22,29$ and 43. Body weight was recorded and evidence of autotomy was looked for on each test day.

\section{Severity of nerve damage}

It is speculated that the severity of sciatic nerve damage influences the development of neuropathic pain ${ }^{16)}$. In our preliminary study, the more the nerve was ligated, the later the onset of pain related-behaviors was and the longer the duration of pain related-behaviors was. So we estimated the severity of nerve damage from motor functions: toe movement; power of plantar flexion; and plantar dorsiflexion. Each behavior was ranked compared with the non- operated left paw: I= no power or movement; II = power or movement severely decreased; III = power or movement slightly decreased ; and $\mathrm{IV}=$ normal power or movement.

\section{Pain related-behaviors}

\section{Paw posture}

The paw posture was observed to speculate the spontaneous pain. The rat was placed in a clear box, and observation of the paw posture was started after a 5 min rest. The paw posture was ranked using the "pain rating" modified from that used in the formalin test ${ }^{2)}$ as follows : $\mathrm{I}=$ the rat licked the paw; II=the whole paw was elevated; III = only the heel was pressed on the floor; IV=only the internal or external edge of the paw was pressed on the floor; $\mathrm{V}=$ the paw rested lightly on the floor; and VI=the paw was pressed normally on the floor. The highest rank of abnormal paw posture (lowest number) during 5 min observation was adopted as a scale.

\section{Sensitivity to touch}

This was measured by determining the withdrawal threshold to touch using calibrated von Frey hairs that ranged between $0.02 \mathrm{~g}$ and $281 \mathrm{~g}$. The rat was placed in a chamber with a metal mesh floor. Each hair was pressed into the mid-plantar area and flexed four times, at a frequency of about $2 / \mathrm{sec}$. For a threshold, we adopted a value at which the rat constantly responded to the successive tests with a paw withdrawal. A von Frey hair below the threshold was used at first, and the strength of hair (in grams) was increased step by step until there were positive responses.

\section{Sensitivity to heat}

This was measured by WLH using Hargreaves' plantar test ${ }^{14)}$. The rat was placed in a clear plastic cage $(18 \times 28 \times 13 \mathrm{~cm})$ upon an elevated floor of window glass. A radiant heat source ( $5 \mathrm{~mm}$ diameter) beneath the glass floor was aimed at the portion of the plantar hind paw where the paw touched with floor, and WLH was measured with a timer responding to beam interruption. The rat was allowed to remove its paw from the heat beam and 20 seconds was the upper limit of allowable exposure. Both hind paws were tested alternatively at $5 \mathrm{~min}$ intervals. Three measurements were taken for each hind 
paw in each test session. Three data per hind paw at each time were used for stastical analysis. This test was done in a temperature controlled area where the glass floor temperature was maintained between 23.0 to $24.0^{\circ} \mathrm{C}$.

\section{Response to mechanical stimulus}

A single prick was given in the mid-plantar area using a sharply pointed metallic rod. The response of the rat was classified as follow: I= absent, if the rat showed no response; II = weak, if the rat responded with a sluggish withdrawal ; $\mathrm{III}=$ normal, if the response was a quick and robust withdrawal ; and IV=exaggerated, if the response was prolonged.

\section{Statistical analysis}

Statistical analysis was performed between bupivacaine and saline groups using repeated measures ANOVA. Within each treatment condition, preoperative baseline data was compared to data for each postopertive day also using repeated measures ANOVA. Non parametric data, such as von Frey hair test, paw posture, response to prick and motor function test were analyzed using either the Mann-Whitney U test or the Friedman test. The criterion for signiflcant difference was $\mathrm{P}<0.05$.

\section{RESULTS}

One rat in the saline group showed no movement of the right foot and no response of the right paw to pinprick for more than three weeks after operation, thus we eliminated this rat from statistical analysis.

There were no significant differences in gain of weight between the saline and bupivacaine or the sham groups. There was no evidence of autotomy in any of the rats ligated. In the sham operation group no pain related-beheviors were observed.

There were no significant differences in severity of nerve damage and all pain relatedbehaviors between bupivacaine and saline groups (Tables 1 4).

\section{Severity of nerve damage}

On post op day 1 no movement of the right toe, and severe to slight decrease in power of right plantar flexion and dorsiflexion were observed in most of rats in bupivacaine and saline groups. The paralysis or decrease in power began to recover by post op day 4 to 8 , and statistically significant occurrence of these motor dysfunctions was observed up to post op day 29 to 43 in both groups.

\section{Pain related-bahaviors \\ Paw posture (Table 1)}

There were no rats which licked or elevated the paw through the observation period. On post op day 1 the paw rested lightly on the floor in almost all rats in both groups. The paw posture gradually recovered. About half of the rats showed the normal paw posture up to post op day 43 in both groups.

\section{Sensitivity to touch (Table 2)}

The median threshold of the right paw to touch decreased from $75.86 \mathrm{~g}$ to $8.51 \mathrm{~g}$ in bupivacaine group and to $5.50 \mathrm{~g}$ in saline group on post op day 1 and gradually recovered in both groups. The significant lowered threshold was observed up to post op day 29 in the bupivacaine group and up to post op day 43 in the saline group. The threshold to touch on the nonoperated left paw did not change in either group.

\section{Sensitivity to heat (Table 3)}

WLH in the right leg was $10.0 \pm 2.3 \mathrm{sec}$ ( mean \pm S.D.) and $9.5 \pm 2.2 \mathrm{sec}$ at baseline which shortened to $6.2 \pm 2.2 \mathrm{sec}$ and $7.0 \pm$ $1.8 \mathrm{sec}$ in the bupivacaine and saline groups respectively on post op day 1 . The shortening was most prominent on post op day 4 and recovered gradually in both groups. The significant shortening was observed up to post op day 43 in both bupivacaine and saline group. A statistically significant shortening to WLH was observed also on the left leg up to post op day 43 and 29 in bupivacaine and saline groups respectively. WLH in the right leg shortened more prominently than that in the left leg from post op day 1 to 15 in both bupivacaine and saline groups.

\section{Response to mechanical stimuli (Table 4)}

An exaggerated response to the right limb to pinprick was observed in most of rats in both groups on post op day 1. The significant high rate of exaggerated response were observed up to post op day 15 in bupivacaine group and up to post op day 29 in saline group. Exaggerated response to pinprick was not observed in the left 
Table 1 Posture of the ligated-side (right) paw following sciatic nerve half ligation

\begin{tabular}{|c|c|c|c|c|c|c|c|c|c|c|c|c|c|c|}
\hline & \multicolumn{7}{|c|}{ Bupivacaine $(n=10)$} & \multicolumn{6}{|c|}{ Saline $(n=9)$} & \multirow[t]{2}{*}{$\begin{array}{l}\text { Bupivacaine } \\
\text { vs. Saline }\end{array}$} \\
\hline & I & II & III & IV & V & VI & & I & II & III & IN & V & VI & \\
\hline pre op. & 0 & 0 & 0 & 0 & 0 & 10 & & 0 & 0 & 0 & 0 & 0 & 9 & N.S. \\
\hline post.1(day) & 0 & 0 & 1 & 0 & 8 & 1 & $\# \#$ & 0 & 0 & 0 & 1 & 8 & $0 \# \#$ & N.S. \\
\hline 4 & 0 & 0 & 0 & 0 & 7 & 3 & \# & 0 & 0 & 0 & 0 & 7 & $2 \#$ & N.S. \\
\hline 8 & 0 & 0 & 0 & 0 & 5 & 5 & & 0 & 0 & 0 & 0 & 5 & 4 & N.S. \\
\hline 15 & 0 & 0 & 0 & 0 & 7 & 3 & $\#$ & 0 & 0 & 0 & 0 & 5 & 4 & N.S. \\
\hline 22 & 0 & 0 & 0 & 0 & 7 & 3 & \# & 0 & 0 & 0 & 0 & 5 & 4 & N.S. \\
\hline 29 & 0 & 0 & 0 & 0 & 6 & 4 & & 0 & 0 & 0 & 0 & 6 & $3 \#$ & N.S. \\
\hline 43 & 0 & 0 & 0 & 0 & 3 & 7 & & 0 & 0 & 0 & 0 & 5 & 4 & N.S. \\
\hline
\end{tabular}

The paw posture was ranked as follows; I; the rat licked the paw, II ; the whole paw was elevated, III ; only the heel was pressed on the floor, IV : only the internal or external edge of the paw was pressed on the floor, $\mathrm{V}$; the paw rested lightly on the floor, VI; the paw was pressed normally on the floor. N.S.; no significance. \# $\mathrm{P}<0.05$ and \#\# $\mathrm{P}<0.01$ versus the preoperative values.

Table 2 Touch withdrawal threshold of the ligatedside (right) paw following sciatic nerve half ligation

\begin{tabular}{rrcccc}
\hline & \multicolumn{2}{c}{$\begin{array}{c}\text { Bupivacaine } \\
(n=10)\end{array}$} & $\begin{array}{c}\text { Saline } \\
(n=9)\end{array}$ & $\begin{array}{c}\text { Bupivacaine } \\
\text { vs. Saline }\end{array}$ \\
\hline pre op & 75.86 & $(28.80-75.86)$ & $75.86(63.88-75.90)$ & N. S. \\
$\begin{array}{r}\text { post } 1 \\
\text { (day) }\end{array}$ & 8.51 & $(5.03-40.52) \#$ & $5.50(3.63-10.00) \# \#$ & N.S. \\
4 & 8.51 & $(3.09-18.05) \#$ & $8.51(1.90-11.75) \#$ & N.S. \\
8 & 5.50 & $(3.23-75.86) \#$ & $5.50(3.63-7.74) \# \#$ & N.S. \\
15 & 3.63 & $(1.48-14.45) \#$ & $5.50(1.90-12.43) \# \#$ & N.S. \\
22 & 5.50 & $(1.48-29.73) \#$ & $2.04(0.35-6.25) \# \#$ & N.S. \\
29 & 3.63 & $(1.41-75.86) \#$ & $1.48(0.35-6.25) \# \#$ & N.S. \\
43 & 11.75 & $(3.23-75.86)$ & $11.75(1.07-29.73) \# \#$ & N.S. \\
\hline
\end{tabular}

The values in the table are median (gram) and interquartile ranges in parentheses. N.S.; no significance. \# $\mathrm{P}<0.05$ and \#\# $\mathrm{P}<0.01$ versus the preoperative values.

limb in both groups.

\section{DISCUSSION}

The neuropathic pain related-behaviors observed in the ligated limb in this study were largely in agreement with those seen by Seltzer et al. ${ }^{21)}$. However in this study the pain relatedbehaviors of the non-ligated limb having been reported by Seltzer et al. were not observed except the hypersensitivity to heat. The pain
Table 3 The comparison of withdrawal latency to heat of ligated (right) and non-ligated (left) legs between bupivacaine and saline group following sciatica nerve half ligation

Ligated: right paw

\begin{tabular}{cccl}
\hline & Bupivacaine $(\mathrm{n}=10)$ & Saline $(\mathrm{n}=9)$ & $\begin{array}{l}\text { Bupivacaine } \\
\text { vs. Saline }\end{array}$ \\
\hline pre op. & $10.0 \pm 2.3$ & $9.5 \pm 2.2 \# \#$ & N.S. \\
post. 1 (day) & $6.2 \pm 2.2 \# \#$ & $7.0 \pm 1.8 \# \#$ & N.S. \\
4 & $5.7 \pm 2.0 \# \#$ & $5.7 \pm 2.0 \# \#$ & N.S. \\
8 & $5.7 \pm 2.3 \# \#$ & $5.8 \pm 2.1 \# \#$ & N.S. \\
15 & $6.3 \pm 1.8 \# \#$ & $5.6 \pm 2.2 \# \#$ & N.S. \\
22 & $7.0 \pm 2.2 \# \#$ & $5.9 \pm 2.0 \# \#$ & N.S. \\
29 & $7.9 \pm 2.3 \# \#$ & $7.0 \pm 2.9 \# \#$ & N.S. \\
43 & $7.2 \pm 2.3 \# \#$ & $7.3 \pm 3.3 \# \#$ & N.S. \\
\hline Non-ligated: right paw & & \\
\hline & Bupivacaine(n=10) & Saline $(\mathrm{n}=9)$ & Bupivacaine \\
pre op. & $9.6 \pm 1.9$ & $8.8 \pm 1.8$ & v. Saline \\
post. 1 (day) & $7.4 \pm 1.4 \# \#$ & $8.5 \pm 1.7$ & N.S. \\
4 & $8.1 \pm 2.0 \# \#$ & $7.9 \pm 1.6 \#$ & N.S. \\
8 & $7.2 \pm 1.5 \# \#$ & $7.2 \pm 1.9 \# \#$ & N.S. \\
15 & $7.4 \pm 1.8 \# \#$ & $7.3 \pm 1.8 \# \#$ & N.S. \\
22 & $7.6 \pm 1.8 \# \#$ & $7.2 \pm 1.4 \# \#$ & N.S. \\
29 & $8.2 \pm 1.5 \# \#$ & $7.2 \pm 1.4 \# \#$ & N.S. \\
43 & $7.6 \pm 1.3 \# \#$ & $8.1 \pm 1.8$ & N.S. \\
\hline
\end{tabular}

The data are shown as mean \pm SD. There were no differences between bupivacaine and saline groups. \# $\mathrm{P}<0.05$, \#\# $\mathrm{P}<0.01$ versus the preoperative values.

Table 4 The responses of the ligated-side (right) paw to pinprick following sciatic nerve half ligation

\begin{tabular}{|c|c|c|c|c|c|c|c|c|c|c|}
\hline & \multicolumn{5}{|c|}{$\begin{array}{l}\text { Bupivacaine } \\
(n=10)\end{array}$} & \multicolumn{4}{|c|}{ Saline $(n=9)$} & \multirow[t]{2}{*}{$\begin{array}{c}\text { Bupivacaine } \\
\text { vs. Saline }\end{array}$} \\
\hline & $I$ & II & III & IV & & I & II & III & IV & \\
\hline pre op. & 0 & 0 & 10 & 0 & & 0 & 0 & 9 & 0 & N.S. \\
\hline post.1(day) & 0 & 1 & 2 & 7 & \# & 0 & 1 & 4 & 4 & N.S. \\
\hline 4 & 0 & 0 & 4 & 6 & \# & 0 & 0 & 2 & $7 \#$ & N.S. \\
\hline 8 & 0 & 0 & 5 & 5 & & 0 & 0 & 2 & $7 \#$ & N.S. \\
\hline 15 & 0 & 0 & 4 & 6 & $\#$ & 0 & 0 & 2 & $7 \#$ & N.S. \\
\hline 22 & 0 & 0 & 5 & 5 & & 0 & 0 & 1 & $8 \# \#$ & N.S. \\
\hline 29 & 0 & 0 & 6 & 4 & & 0 & 0 & 2 & $7 \#$ & N.S. \\
\hline 43 & 0 & 0 & 7 & 3 & & 0 & 0 & 4 & 5 & N.S. \\
\hline
\end{tabular}

The response of the paw was ranked as follows; I ; absent, if the rat showed no response: II ; weak, if the rat responded with a sluggish withdrawal; II ; normal, if the response was quick; IV; exaggerated, if the response was prolonged. N.S.; no significance. \# $\mathrm{P}<0.05$ and \#\# $\mathrm{P}<0.01$ versus the preoperative values. 
related-behaviors of the non-ligated limb supposedly depend on central hypersensitivity which is produced by inputs from a peripheral injury ${ }^{6)}$. The different effect of ligation on the non-ligated limb might be the results of strain difference; Sprague-Dawley rats in our study, on the other hand Sabra rats in Selzer group. Our results are similar to those reported by other investigators that have used PL model on Sprague-Dawley rats $^{4,18)}$.

In this study, there was no prophylactic effect of prior nerve block on any pain relatedbehaviors up to 43 days after ligation. This strongly suggests that prior nerve block has no prophylactic effect on the development of neuropathic pain in PL model.

Abnormal afferent impulses following nerve injury is studied mainly in sciatic nerve transection model, the model of phantom limb pain or anesthesia dolorosa. Massive afferent impulses are generated during and several seconds after nerve injury ${ }^{25)}$, followed by the ectopic discharge generated in the damaged nerves or dorsal ganglion cells within days ${ }^{26)}$. In partial nerve damage like PL model ectopic discharge presumably follows soon after the injury discharge, or injury discharge and ectopic discharge is a continuum. This speculation may be substantiated by the fact that in PL model pain related-behaviors were observed soon after the nerve injury ${ }^{19}$.

The hypothesis that long-lasting pain results from the neuronal plasticity triggered by afferent input from the periphery easily suggests that the more and/or the longer the injury and ectopic discharge is, the more severe pain develops ${ }^{6,11,27)}$. We can not deny the possibility that the failure of preventing the development of nouropathic pain in this study results from the short duration of the nerve block.

From this and other studies, it can be said that there is no effect of prior nerve block on the development of pain related-behaviors in PL model $^{10)}$ and some effects in CCI model ${ }^{10,23,29)}$. Several studies in the sciatic nerve transection model ${ }^{17,22,26)}$ show that prior nerve block reduced the severity and delayed the average time of onset of autotomy. These differences among models suggest that the importance of injury discharge in the development of neuropathic pain differs among models, and that different mechanisms operate in the development of neuropathic pain in different models.

\section{Acknowledgement}

The authors wish to thank to Lewis, B.K. for technical assistance.

\section{REFERENCES}

1) Abbott, F. V. and Melzack, R., Dissociation of the mechanisms of stimulation-produced analgesia in tests of tonic and phase pain. In : J. J. Bonica, U. L. F. Lindblom and A. Iggo (Eds.), Advances in Pain Research and Therapy, Vol. 5, Raven Press, New York, 1983, pp. 401-409.

2) Attal, N., Jazat, F., Kayser, V. and Guilbaud, G., Further evidence for pain-related behaviors in a model of unilateral peripheral mononeuropathy, Pain, 41 (1990) 235-251.

3) Bach, S., Noreng, M. F. and Tjëllden, N. U., Phantom limb pain in amputees during the first 12 months following limb amputation, after preoperative lumbar epidural blockade, Pain, 33 (1988) 297-301.

4) Behbehani, M. M. and Dollberg-Stolik, O., Partial sciatic nerve ligation results in an enlargement of the receptive field and enhancement of the response of dorsal horn neurons to noxious stimulation by an adenosine agonist, Pain, 58 (1994) 421-428

5) Bennett, G. J. and Xie, Y. K., A peripheral mononeuropathy in rat that produces disorders of pain sensation like those seen in man, Pain, $\mathbf{3 3}$ (1988) 87-107.

6) Coderre, T., Katz, J., Vaccarino, A. L. and Melzack, R., Contribution of central neuroplasticity to pathological pain: review of clinical and experimental evidence, Pain, 52 (1993) 259285.

7) Conacher, I. D., Therapists and therapies for post-thoracotomy neuralgia, Pain, 48 (1992) 409-412.

8) Davar, G., Hama, A., Deykin, A., Vos, B. and Maciewicz, R., MK-801 blocks the development of thermal hyperalgesia in a rat model for experimental painful neuropathy, Brain Res., 553 (1991) 327-330.

9) Devor, M., Neuropathic pain and injured nerve : peripheral mechanisms, Br. Med. Bull., 47 
(1991) 619-630.

10) Dougherty, P. M., Garrison, C. J. and Carlton, S. M., Differential influence of local anesthetic upon models of experimentally induced peripheral mononeuropathy in the rat, Brain Res., 570 (1992) 109-115.

11) Dubner, R. and Ruda, M. A., Activity-dependent neuronal plasticity following tissue injury and inflammation, Trends Neurosci., 15 (1992) 96-103.

12) Gonzalez-Darder, J. M., Barber, J. and Abellan, M. J., Effects of prior anaesthesia on autotomy following sciatic transection in rats, Pain, 24 (1986) 87-91.

13) Goto, T., Marota, J. J. A. and Crosby, G., Nitrous oxide induces preemptive analgesia in the rat that is antagonized by halothane, Anesthesiology, 80 (1994) 409-416.

14) Hargreaves, K., Dubner, R., Brown, F., Flores, C. and Joris, J., A new and sensitive method for measuring thermal nociception in cutaneous hyperalgesia, Pain, 32 (1988) 77-88.

15) Hirata, H., Pataky, A., Kajander, K., LaMotte, R. H., Collins, J. G., A model of peripheral mononeuropahty in the rat, Pain, 42 (1990) 253254.

16) Hylden, J. L. K., Nahin, R. L., Humphrey, E., Seltzer, Z. and Dubner, R., An animal model of hyperalgesia: partial sciatic nerve lesion, Pain Suppl., 4 (1987) S274.

17) Katz, J., Vaccarino, A. L., Coderre, T. J. and Melzack, R., Injury prior to neurectomy alters the pattern of autotomy in rats; Behavioral evidence of central neural plasticity, Ansethesiology, 75 (1991) 876-883.

18) Kim, S. H. and Chung, J. M., An experimental model for peripheral neuropathy produced by segmental spinal nerve ligation in the rat, Pain, 50 (1992) 355-363.

19) Mao, J., Price, D. D., Mayer, D. J., Lu, J. and Hayes, R. L., Intrathecal MK-801 and local nerve anesthesia synergistically reduce nociceptive behaviors in rats with experimental peripheral mononeuropathy, Brain Res., 576 (1992) 254-262.

20) Mao, J., Price, D. D., Mayer, D. J. and Hayes, R. L., Central nervous system mechanisms of neuropathic pain : new treatment by ganglioside. In :
L. Vecchiet, D. Albe-Fessard and U. Lindblom (Eds.), New Trends in Referred Pain and Hyperalgesia, Elsevier, New York, 1993, pp. 259-272.

21) Seltzer, Z., Dubner, R. and Shir, Y., A novel behavioral model of neuropathic pain disorders produced in rats by partial sciatic nerve injury, Pain, 43 (1990) 205-218.

22) Seltzer, Z., Beilin, B., Ginzburg, R., Paran, Y. and Shimko, T., The role of injury discharge in the induction of neuropathic pain behavior in rats, Pain, 46 (1991) 327-336.

23) Sotgiu, M. L., Castagna, A., Lacerenza, M. and Marchettini, P., Pre-injury lidocaine treatment prevents thermal hyperalgesia and cutaneous thermal abnormalities in a rat model of peripheral neuropathy, Pain, 61 (1995) 3-10.

24) Tasker, R. R., Deafferentation. In : P. D. Wall and R. Melzack (Eds.), Textbook of Pain, Churchill Livingstone, New York, 1984, pp. 119132.

25) Wall, P. D., Waxman, S. and Basbaum, A. I., Ongoing activity in peripheral nerve: injury discharge, Exp. Neurol., 45 (1974) 576-589.

26) Wall, P. D. and Devor, M., Sensory afferent impulses originate from dorsal root ganglia as well as from the peripherary in normal and nerve injured rats, Pain, 17 (1983) 321-339.

27) Wall, P. D., Neuropathic pain and injured nerve: Central mechanisms, Br. Med. Bull., 47 (1991) 631-643.

28) Woolf, C. J. and Thompson, S. W. N., The induction and maintenance of central sensitization is dependent on $\mathrm{N}$-methyl-D-aspartic acid receptor activation; implications for the treatment of post-injury pain hypersensitivity states, Pain, 44 (1991) 293-299.

29) Yamamoto, T., Shimoyama, N. and Mizuguchi, $\mathrm{T}$., Role of the injury discharge in the development of thermal hyperesthesia after sciatic nerve constriction injury in the rat, Anesthesiology, 79 (1993) 993-1002.

Address for correspondence: Takumi Nagaro, M.D., Department of Anesthesiology \& Resuscitology, Ehime University School of Medicine, Shigenobu, Ehime 79102, Japan.

Tel : +81-89-960-5383

Fax : +81-89-964-7494 